\title{
A Comparison of Symptoms, Functional Status and Quality of Life by the Survivor Stages of Pancreatic Cancer Patients
}

\author{
Jisun Lee \\ Professor, Department of Nursing, Honam University, Republic of Korea
}

\begin{abstract}
Background/Objectives: The purpose of this study was to identify the difference of symptoms, functional status and quality of life (QOL) according to the survival stage of pancreatic cancer patients.

Method/Statistical analysis: Descriptive, cross-sectional design. A sample of 139 patients was surveyed. The participants were as patients who were diagnosed with pancreatic cancer in Republic of Korea. The data collected was analyzed with descriptive statistics.

Findings: Nausea and vomiting symptoms were significantly higher in acute survival stage than in extended survival stage and insomnia and diarrhea were significantly higher in extended survival stage than in acute survival stage functional status and QOL were significantly higher in extended survival stage than in acute survival stage.

Improvements/Applications: The differences in symptoms, functional status and QOL by the survival stage of pancreatic cancer patients were grasped and the empirical evidence was given for performing differentiated nursing interventions by each survival stage.
\end{abstract}

Keywords: Pancreatic cancer. Survival stages, Symptoms, Functional status, Quality of life.

\section{Introduction}

Pancreatic cancer is one of the major diseases that rank fifth in cancer mortality rates in Republic of Korea, the 5-year relative survival rate of pancreatic cancer is $11.1 \%$, which can be found as very low compared to the overall cancer survival rate of $70.6 \%^{[1]}$. Pancreatic cancer which is a low survival rate, nursing that prioritizes the quality of life (QOL) is required not from the last day to a couple of weeks, but from the day of diagnosis ${ }^{[2]}$. Most of the patients with pancreatic cancer would be threatened their QOL by unfavorable prognosis ${ }^{[3,4,5]}$.

QOL is a subjective well-being condition that individuals feel about their lives and it is raising its

\footnotetext{
Corresponding Author:

Jisun Lee

Professor, Department of Nursing, Honam University,

Republic of Korea

e-mail: leezggo@naver.com
}

head as an evaluation index of the treatment of cancer ${ }^{[6]}$. To maintain the highest level of well-being of the subject which is the main purpose of nursing and help, identify the factors affecting the QOL of pancreatic cancer patients and develop ways to mediate them is cancer patients experience a variety of symptoms and changes in functional status as they undergo physical, psychological and cognitive changes in the treatment and progress of the disease, among them, the symptoms play an important role in functional conditions and $\mathrm{QOL}^{[7]}$. The functional status of patients diagnosed with pancreatic cancer is reported to be very low compared to other cancer diseases ${ }^{[8]}$, so efforts should be preceded to verify the patient's functional status and to understand the nursing needs of those diagnosed clearly.

Mullen ${ }^{[9]}$ classified the cancer survival stage into three stages based on changes in cancer condition and treatment process after cancer was diagnosed and argued that the required intervention method for each stage was different. These survival stages are a complex process 
that occurs continuously and dynamically in the begins with the diagnosis of the cancer ${ }^{[10]}$. Therefore, assessing and interpreting the symptoms, functional status and QOL of survivors at each stage and differentiated arbitration by each survival stage should be applied.

The purpose of this study was to identify the difference of symptoms, functional status and QOL according to the survival stage of pancreatic cancer patients and to use it as evidence for developing differentiated nursing intervention according to the characteristics of survival stage.

\section{Method}

Research Design: This study is a descriptive-cross sectional study.

Research Subjects: The participants were as patients who were diagnosed with pancreatic cancer and visiting outpatient departments of one general hospital in Republic of Korea for treatment and further care. The inclusion criteria for this study were (a)adults $>20$ years old, (b) diagnosed patient with pancreatic cancer and (c) patients capable of understanding the contents of the questionnaire and responding directly to the questionnaire. The exclusion criteria for this study were (a) patients with psychiatric anamnesis or those receiving medication, (b) non-protopathic pancreatic cancer and (c) serious comorbid diseases requiring treatment other than pancreatic cancer.

The sample size of this study was calculated as a total of 126 people by using the $G^{*}$ power program when it set as t-test significance level $(\alpha)=0.05$, effect size $=0.5$ and verification power $(1-\beta)=80^{[11]}$.

Among data from 140 people which considered the dropout rate of $10 \% .139$ people were used as final analysis data except for one questionnaire which was insufficiently responded. As a result of the classification by survival stage, It was 100 people in the acute survival stage, 31 in the extended survival stage and 8 in the permanent survival stage that, the number of subjects in the permanent survival stage was not enough so the survival stage was reclassified to 2 stages by including the permanent survival stage in the extended survival stage based on previous research ${ }^{[11]}$. Finally, the patients were divided into 100 people in acute survival stages that less than 2 years after diagnosis of pancreatic cancer and 39 people in extended survival stages that more than 2 years after diagnosis of pancreatic cancer.

\section{Measurement:}

Symptoms: In this study used the sub territory of the QOL related to symptoms among the Korean version tools of EORTC QLQ-C30 (version 3). It is composed of 4 point scale and calculated from 0 to 100 points as perfect score according to the scoring instructions of the tool. Lower scores indicate better symptoms. At the time of development, it was Cronbach's $\alpha=85$ and in this study, it was Cronbach's $\alpha=.87$.

Functional status: In this study, using the sub territory of the QOL related functional status among the Korean version tools of EORTC QLQ-C30 (version 3). There are totals of 15 items, including five items of physical function, two items of role function, two items of cognitive function, two items of emotional function, two items of social function. It is composed of 4 point scale and calculated from 0 to 100 points as perfect score according to the scoring instructions of the tool. Higher scores indicate better functional status. At the time of development, it was Cronbach's $\alpha=90$ and in this study, it was Cronbach's $\alpha=.91$.

Quality of life: In this study used the Overall QOL among the Korean version tools of EORTC QLQ-C30 (version 3). There are totals of 2 items including 1 item of general health condition and 1 item of QOL and It is 7 point scale and calculated from 0 to 100 points as perfect score according to the scoring instructions of the tool, which means that the higher the score, the better the QOL. At the time of development, it was Cronbach's $\alpha=84$ and in this study, it was Cronbach's $\alpha=.86$.

Data Collection: The data collection of the study was conducted from June 15, 2018 to August 30. The list of patients visiting the outpatient department of a general hospital. The survey was conducted by including those who voluntarily agreed to participate in the study after the researcher explained by orally and in writing of the purpose and procedures of the study to the selected person, then conducted through the way of the subject directly filling out the questionnaire.

Data Analysis: The collected data were analyzed using the SPSS/WIN 25.0 program as follows. The differences in general characteristics, disease-related characteristics, symptoms, functional status and QOL were analyzed with $\chi 2$ test and t-test. Substantial missing data, one questionnaire were excluded from analysis. p-values $<0.05$ were considered significant. 


\section{Result and Discussion}

The differences in general characteristics and disease-related characteristics: The number of people in acute survival stages was the largest at 100 (71.9\%) and the number of people in extended survival stages was $39(28.1 \%)$. As a general characteristic, the largest number of the gender was men as $100(71.9 \%)$ and the largest number of age was between 56 and 65 as 59 (42.4\%). In disease-related characteristics, the largest number of pancreatic cancer locations was in the head of the pancreatic as 89 people $(64.0 \%)$ and metastasized cases as 75 people (54.7\%). As for the treatment method, It was shown that 104 people $(74.8 \%)$ took surgical treatment and 76 people (54.7\%) took chemotherapy and 123 people ( $88.5 \%$ ) did not take radiation therapy. There was no significant difference between the acute survival stage and the extended survival stage according to the analysis result of the difference in general characteristics and disease-related characteristics according to the survival stage of pancreatic cancer patients.

Differences in symptoms, functional status and QOL: [Table 1] shows the differences in symptoms, functional status and QOL in pancreatic cancer patients. Nausea and vomiting were significantly higher in acute survival stage as $56.38 \pm 13.15$ than in extended survival stage as $45.82 \pm 10.91(\mathrm{t}=-4.45, p<.001)$ and insomnia was significantly higher in extended survival stage as $52.59 \pm 11.72$ than in acute survival stage as $42.59 \pm 11.72(\mathrm{t}=-4.70, p<.001)$ and the diarrhea was significantly higher in the extended survival stage as $55.51 \pm 7.40$ than the acute survival stage $50.60 \pm 4.87(\mathrm{t}=-$ $4.70, p<.001)$. As a result of analyzing the difference of functional status by survival stage, the physical function was significantly higher in extended survival stage as $57.69 \pm 8.25$ than in acute survival stage of $53.47 \pm 9.99$

$(\mathrm{t}=-2.55, p=.013)$, the role function was also in extended survival stage as $48.8 \pm 13.47$ than in acute survival stage of $42.79 \pm 10.62(\mathrm{t}=-2.50, p=.015)$ and the cognitive function was significantly higher in extended survival stage as $49.01 \pm 13.27$ than in acute survival stage as $40.46 \pm 0.116(\mathrm{t}=-3.63, p=.001)$. As a result of analyzing the difference in the QOL in patients with pancreatic cancer showed that the extended survival stage was $55.40 \pm 6.35$, significantly higher than the acute survival stage of $51.54 \pm 4.67(\mathrm{t}=-3.45, p=.001)$.

\section{Discussion}

This study was conducted to identify symptoms, functional status and differences in QOL according to the survival stages of pancreatic cancer patients.

In this study, as a result of looking into the difference of symptoms according to the survival stage, was found that in the acute survival stage was more complain of nausea and vomiting symptoms than in the extended survival stage and in the extended survival stage more severe insomnia and diarrhea symptoms than in the acute survival stage. Result of this was similar to a study that reported in the Cohort study of 564 pancreatic cancer survivors that pancreatic cancer survivors complained of symptoms such as nausea, vomiting, insomnia, fatigue, pain and loss of appetite ${ }^{[12]}$. In particular, the symptoms of nausea and vomiting are accompanied by symptoms of appetite loss and based on studies that report that $75 \%$ of patients with pancreatic cancer complain of appetite loss ${ }^{[13]}$, it is necessary to evaluate the status of food intake and nutrition absorption and to make efforts for continuous management in order to confirm the nutritional status as well as the intervention for relieving symptoms according to the survival stage of pancreatic cancer patients. Especially in the case of undernourished cancer patients, symptoms such as electrolyte imbalance, infection and fatigue were accompanied, consequentially a negative impact on the survival rate and QOL of cancer patients appeared ${ }^{[12]}$. Hence, evaluate the nutritional status of the individual and to organize a nutrition program to so that the necessary nutrients can be properly consumed in order to control their symptoms and improve nutrition intake and efforts that continuously watch and support the dietary intake and nutritional state are required.

Meanwhile, the symptoms of cancer patients are multiple symptoms at the same time, especially for pancreatic cancer patients, there are $5 \sim 10$ symptoms exist at the same time and it appeared that they are interrelated ${ }^{[14]}$. As such, it is necessary to verify whether there is a difference in the survival stage of pancreatic cancer patients with the symptom cluster in which exists two or more symptoms at the same time is different in the survival stage of pancreatic cancer patient.

The functional status according to the survival stage was significantly higher in physical function, role function and cognitive function in the extended survival stage than in the acute survival stage. These results can be predicted as the patient would be faced anxiety and shocked by sudden diagnosis in the early stages of diagnosis, but with the passage of time, they adapt and handle disease and treatment and become better functional status. 
Table 1. Differences in symptoms, functional status and QOL in pancreatic cancer patients

\begin{tabular}{|c|c|c|c|c|c|c|}
\hline \multirow{2}{*}{ Variables } & \multirow{2}{*}{ Categories } & \multirow{2}{*}{$\mathbf{M} \pm \mathbf{S D}$} & $\operatorname{ASS}^{\dagger}(n=100)$ & $\operatorname{ESS}^{*}(\mathbf{n}=39)$ & \multirow{2}{*}{$\mathbf{t}$} & \multirow{2}{*}{$\mathbf{P}$} \\
\hline & & & $\mathbf{M} \pm \mathbf{S D}$ & $\mathbf{M} \pm \mathbf{S D}$ & & \\
\hline \multirow[t]{9}{*}{ Symptoms } & Fatigue & $55.29 \pm 11.23$ & $54.17 \pm 11.19$ & $58.16 \pm 10.11$ & -1.90 & .059 \\
\hline & Nausea/vomiting & $48.78 \pm 12.48$ & $56.38 \pm 13.15$ & $45.82 \pm 10.91$ & -4.45 & $<.001$ \\
\hline & Pain & $49.26 \pm 11.77$ & $54.53 \pm 11.67$ & $47.21 \pm 10.92$ & -4.14 & .061 \\
\hline & Dyspnea & $46.08 \pm 10.15$ & $43.71 \pm 10.92$ & $46.94 \pm 7.94$ & -.55 & .586 \\
\hline & Insomnia & $45.58 \pm 10.63$ & $42.85 \pm 8.83$ & $52.59 \pm 11.72$ & -4.70 & $<.001$ \\
\hline & Appetite loss & $45.34 \pm 10.11$ & $44.46 \pm 10.16$ & $47.15 \pm 8.31$ & -4.00 & .052 \\
\hline & Constipati-on & $59.54 \pm 6.49$ & $59.97 \pm 7.22$ & $58.43 \pm 3.92$ & 1.61 & .109 \\
\hline & Diarrhea & $51.98 \pm 6.09$ & $50.60 \pm 4.87$ & $55.51 \pm 7.40$ & -3.83 & $<.001$ \\
\hline & Financial difficulties & $54.04 \pm 10.68$ & $54.17 \pm 11.49$ & $53.72 \pm 8.39$ & .26 & .799 \\
\hline \multicolumn{2}{|c|}{ Total symptom score } & $50.21 \pm 5.80$ & $48.85 \pm 5.10$ & $53.69 \pm 6.10$ & -4.39 & $<.001$ \\
\hline \multirow{5}{*}{ Functional status } & Physical & $54.65 \pm 9.70$ & $53.47 \pm 9.99$ & $57.69 \pm 8.25$ & -2.55 & .013 \\
\hline & Role & $44.48 \pm 11.76$ & $42.79 \pm 10.62$ & $48.8 \pm 13.47$ & -2.50 & .015 \\
\hline & Cognitive & $42.86 \pm 11.72$ & $40.46 \pm 10.16$ & $49.01 \pm 13.27$ & -3.63 & .001 \\
\hline & Emotion & $60.01 \pm 6.92$ & $59.97 \pm 7.22$ & $60.12 \pm 6.17$ & -0.11 & .911 \\
\hline & Social & $59.77 \pm 9.84$ & $60.52 \pm 9.18$ & $57.83 \pm 11.25$ & 1.45 & .148 \\
\hline \multicolumn{2}{|c|}{ Total functional status score } & $51.24 \pm 4.61$ & $51.44 \pm 4.65$ & $50.72 \pm 4.51$ & .843 & .402 \\
\hline \multicolumn{2}{|l|}{ Quality of life } & $52.63 \pm 6.45$ & $51.54 \pm 4.67$ & $55.40 \pm 6.35$ & -3.45 & .001 \\
\hline
\end{tabular}

${ }^{\dagger}$ Acute survival stage; ${ }^{\star E x t e n d e d ~ s u r v i v a l ~ s t a g e ~}$

Meanwhile, In the study of Carrato et al. ${ }^{[2]}$ reported that patients who took pancreatic cancer resection showed the longest average survival rate in the $11 \sim 25.7$ months range and that patients who took chemotherapy, radiation therapy, palliative surgery or exploratory laparotomy had an average survival period of between $2 \sim 8.1$ months and 1.1 months for patients who took no surgery or active treatment. Compared the prognosis and survival rates of these pancreatic cancers with the results of this study, it is predicted that the overall functional status was measured as low due to the poor prognosis in the acute survival stage and numerous survivors who are focused on treatment.

This study confirmed the contents what Mullen ${ }^{[9]}$ argued that the intervention method required by the cancer survival stage was different. In addition, the differences in symptoms, functional status and QOL by the survival stage of pancreatic cancer patients were grasped and the theoretical basis was given for performing differentiated nursing interventions by each survival stage.

\section{Conclusion}

This study aims to identify the differences in symptoms, functional status and QOL depending on the survival stage of pancreatic cancer patients and to utilize them as basic data for the development of differentiated nursing interventions program considering the characteristics of each survival stage.

The study result has shown that in the symptoms of pancreatic cancer patients by survival stage, symptoms of nausea and vomiting in patients with pancreatic cancer are significantly higher in the acute survival stage than in the extended survival stage and that insomnia and diarrhea in the extended survival stage are significantly higher than in the acute survival stage. Functional status (physical function, role function and cognitive function) and QOL were significantly higher in the extended survival stage than in the acute survival stage. Based on the above results of this study, the following suggestions are proposed. As a result of this study, although there were differences in symptoms, functional status and QOL according to the survival stage, it is difficult to 
generalize the study results because the clinical stage, metastasis or not and treatment conditions of pancreatic cancer survivors who configured for each survival stage, are not identical. Therefore, further studies that collect and compare the same subjects need to be conducted.

Ethical Clearance: IRB 2018-06-02

\section{Conflict of Interest: Nil}

Source of Funding: Self

\section{References}

1. National Cancer Information Center. 5-year relative survival rate. [Internet]. 2019 [updated 2019 Feb; cited 2019 Sep 10]. Available from: https:// www.cancer.go.kr/lay1/S1T648C650/contents.do. (webiste)

2. Carrato A, Falcone A, Ducreux, M, Valle JW, Parnaby A, Djazouli K., ... \& Parthenaki I. A systematic review of the burden of pancreatic cancer in Europe: real-world impact on survival, quality of life and costs. J Gastrointest Cancer. 2015 Sep; 46(3), 201-211. DOI: http://dx.doi. org/10.1007/s12029-015-9724-1

3. Bye A, Jordhøy MS, Skjegstad G, Ledsaak O, Iversen P O, \& Hjermstad MJ. Symptoms in advanced pancreatic cancer are of importance for energy intake. Support CareCancer. 2012 June; 21(1), 219-227.

4. Zabernigg A, Giesinger JM, Pall G, Gamper EM, Gattringer K, Wintner LM, ... \& Holzner B. Quality of life across chemotherapy lines in patients with cancers of the pancreas and biliary tract. BMC cancer. 2012 Sep;12(1), 390. DOI: https://doi. org/10.1186/1471-2407-12-390.

5. Kim GD, Jang HJ. Effects of Pain, Sleep Disturbance and Fatigue on the Quality of Life in Patients with Pancreatic Cancer Undergoing Chemotherapy. Asian Oncol Nurs. 2012 Jun; 12(2), 117-124.

6. Kim HW, Choi KS. Structural Equation Modelingon Quality of Life in Pre-dialysis Patients with Chronic Kidney Disease. J Korean Acad Nurs. 2012 42(5), 699-708. $\square \square$ ?

7. Dodd M, Janson S, Facione N, Faucett J, Froelicher ES, Humphreys J, ... \& Taylor D. Advancing the science of symptom management. J Adv Nurs. 2008 July; 33(5), 668-676. https://doi.org/10.1046/ j.1365-2648.2001.01697.x.

8. Hwang HK. Quality of life after total pancreatectomy (Doctoral dissertation). Yonsei University, Seoul. Republic of Korea. 2014

9. Mullan F. Seasons of survival: reflections of a physician with cancer. N Engl J Med.1985 July; 313, 270-273. DOI: https://doi.org/10.1056/ NEJM198507253130421.

10. Zebrack, BJ. Cancer survivor identity and quality of life.Cancer Pract, 2001 Dec; 8(5), 238-242. DOI: https://doi.org/10.1046/j.1523-5394.2000.85004. $\mathrm{x}$.

11. Chae YJ \& Park JS. A Comparison of Symptoms, Depression and Quality of Life according to Stages of Survivorship in Lung Cancer Patients. Asian Oncol Nurs. 2017 June; 17(2), 79-86. DOI: https:// doi.org/10.5388/aon.2017.17.2.79.

12. Gupta D, Lis CG, \& Grutsch JF. The European organization for research and treatment of cancer quality of life questionnaire: implications for prognosis in pancreatic cancer. Int $\mathrm{J}$ Gastrointest Cancer, 2006 Sep; 37(2-3), 65-73. DOI: http:// dx.doi.org/10.1007/s12029-007-0001-9.

13. Conroy T, Uwer L, \& Deblock M. Health-related quality-of-life assessment in gastrointestinal cancer: are results relevant for clinical practice? Curr Opin Oncol, 2007Jul; 19(4), 401-406. DOI: https://doi.org/10.1097/CCO.0b013e32816f7704.

14. Yeo TP, Burrell SA, Sauter PK, Kennedy EP, Lavu H, Leiby BE, \& Yeo CJ. A progressive postresection walking program significantly improves fatigue and health-related quality of life in pancreas and periampullary cancer patients. $\mathrm{J}$ Am Coll Surg, 2012 Apr; 214(4), 463-475. DOI: https://doi.org/10.1016/j.jamcollsurg.2011.12.017. 